\title{
THE EFFECTS OF RADIATION AND HEAT GENERATION ON MAGNETOHYDRODYNAMIC(MHD) NATURAL Convection Flow Along a Vertical Flat Plate in PRESENCE OF VISCOUS DISSIPATION
}

\author{
Mohammad Mokaddes Ali ${ }^{1}$, Rowsanara Akhter ${ }^{2}$, NHM. A. Azim ${ }^{3}$ and M. A. Maleque ${ }^{4}$ \\ ${ }^{1}$ Faculty of Life Science, Mawlana Bhashani Science and Technology University, Tangail-1902, \\ Bangladesh, \\ ${ }^{2,4}$ Department of Mathematics, Bangladesh University of Engineering and Technology, Dhaka- \\ 1000, Bangladesh \\ ${ }^{3}$ School of Business Studies, Southeast University, Dhaka-1000, Bangladesh
}

E-mail:_mmokaddesali@yahoo.com, rakhter309@gmail,amaleque@math.buet.ac.bd,nhmarif1@gmail.com

\begin{abstract}
This article investigates the effects of radiation and heat generation on magnetohydrodynamic(MHD) natural convection flow of an incompressible viscous electrically conducting fluid along a vertically placed flat plate in presence of viscous dissipation and heat conduction. Appropriate transformations were employed to transform governing equations of this flow into dimensionless form and then solved using the implicit finite difference method with Keller box scheme. The resulting numerical solutions of transformed governing equations are presented graphically in terms of velocity profile, temperature distribution, skin friction coefficient and surface temperature and the effects of magnetic parameter $(M)$, radiation parameter $(R)$, Prandtl number (Pr) and heat generation parameter $(Q)$ and viscous dissipation parameter (N) on the flow have been studied with the help of graphs.
\end{abstract}

Keywords: Radiation, Heat Generation Parameter, Viscous Dissipation Parameter, MHD, Finite Difference Method, Vertical Flat Plate.

\section{Nomenclature \\ $b \quad$ Plate thickness \\ $C_{f x} \quad$ Local skin friction coefficient \\ $C_{p} \quad$ Specific heat at constant pressure}

$\begin{array}{ll}f & \text { Dimensionless stream function } \\ g & \text { Acceleration due to gravity } \\ G_{r} & \text { Grashof number } \\ h & \text { Dimensionless temperature } \\ H_{0} & \text { Strength of magnetic field } \\ k_{f}, k_{s} & \text { Fluid and solid thermal } \\ l & \text { conductivities } \\ M & \text { Length of the plate } \\ N & \text { Magnetic parameter } \\ p & \text { Viscous dissipation parameter } \\ \operatorname{Pr} & \text { Conjugate conduction parameter } \\ q_{w} & \text { Prandtl number } \\ Q & \text { Heat flux } \\ R & \text { Reat generation parameter } \\ T_{b} & \text { Temperature at outside surface of the } \\ T_{f} & \text { plate } \\ T_{w} & \text { Temperature of the fluid } \\ T_{\infty} & \text { Average temperature of porous plate } \\ \bar{u}, \bar{v} & \text { Temperature of the ambient fluid } \\ u, v & \text { Velocity components } \\ \bar{x}, \bar{y} & \text { Dimensionless velocity components } \\ x, y & \text { Cartesian co-ordinates } \\ \beta & \text { Dimensionless Cartesian co-ordinate } \\ \eta & \text { Coefficient of thermal expansion } \\ \theta & \text { Dimensionless similarity variable } \\ \mu & \text { Dimensionless temperature } \\ & \text { Viscosity of the fluid } \\ & \end{array}$




$\begin{array}{ll}\nu & \text { Kinematic viscosity } \\ \rho & \text { Density of the fluid } \\ \sigma & \text { Electrical conductivity } \\ \tau_{w} & \text { Shearing stress } \\ \psi & \text { Stream function }\end{array}$

\section{Introduction}

The natural convection about a heated vertical flat plate has received a great deal of attention due to its potential applications. In addition, MHD natural convection flow has significant applications in the field of stellar and planetary magnetospheres, aeronautics, chemical engineering and electronics. Considering of its importance, these flow have been studied several research groups [1-3]. As the engineering processes closely related to temperature, accordingly radiation heat transfer has significant influence on engineering. Due to its wide applications in space technology such as cosmical flight, aerodynamics rocket, propulsion systems, plasma physics, spacecraft re-entry aerodynamics and at high operators' temperature a lot of researchers studied the effect of radiation on MHD free convection flow. Takhar and Soundalgekar [4] studied the effect of radiation on MHD free convection flow of a gas past a sami-infinite vertical plate using the Cogley-vincenti-Giles equilibrium model. The problem of natural convectionradiation interaction on boundary layer flow with Rosscland diffusion approximation along a vertical thin cylinder has been investigated by Hossain and Alim [5]. Radiation effect on free convection flow of fluid from a porous vertical plate was studied by Hossain et al. [6]. Thermal radiation and buoyancy effects on MHD free convection heat generating flow over an accelerating permeable surface with temperature- dependent viscosity studied by Seddeek [7]. Abdel-naby et al. [8] studied the radiation effects on MHD unsteady free convection flow over a vertical plate with variable surface temperature. Moreover, heat generation effects in moving fluids is important in problems dealing with the chemical reactions and those concerned with dissociating fluids such as heat generation are resistance heating in wires, exothermic chemical reactions in a solid and nuclear reactions in nuclear fuel rods where electrical, chemical and nuclear energies are converted to heat. Furthermore, the viscous dissipation of heat in the natural convection flow is important when the flow field is of extreme size or in high gravitational field. Experimental and theoretical works on heat generation effects and viscous dissipation effects have been done extensively [9-13] but, to the best of our knowledge, no one has considered combined effect of radiation, heat generation and viscous dissipation under the process of steady natural convection flow. So, the objective of the present work is to study the effect of radiation, heat generation and viscous dissipation on MHD natural convection flow of an incompressible, viscous and electrically conducting fluid along a vertical flat plate under the influence of transverse magnetic field in presence of heat conduction. The governing partial differential equations are reduced to locally non-similar partial differential forms by using appropriate transformations. The transformed boundary layer equations are solved numerically adopting implicit finite difference method together with Keller Box Scheme technique [14, 15]. Here, the assumption is focused on the evaluation of the surface shear stress in terms of local skin friction, surface temperature, velocity profiles and temperature distribution for selected values of parameters consisting magnetic parameter $M$ , radiation parameter $R$, Prandtl number $P r$, heat generation parameter $Q$ and viscous dissipation parameter $N$.

\section{Mathematical Analysis}

Let us consider a steady, free convection boundary layer flow of an incompressible and electrically conducting fluid along a vertical flat plate of length $l$ and thickness $b$. The temperature $T_{b}$ at the outer surface of the plate is considered constant and greater than the temperature $T_{\infty}$ of the ambient fluid. The effect of radiation, heat generation and viscous dissipation in the flow region and conduction with in the plate are taken into account in this analysis. An uniform magnetic field of strength $H_{0}$ is imposed along the $\bar{y}$-axis. The flow configuration and the coordinates system are shown in Fig.-1. 


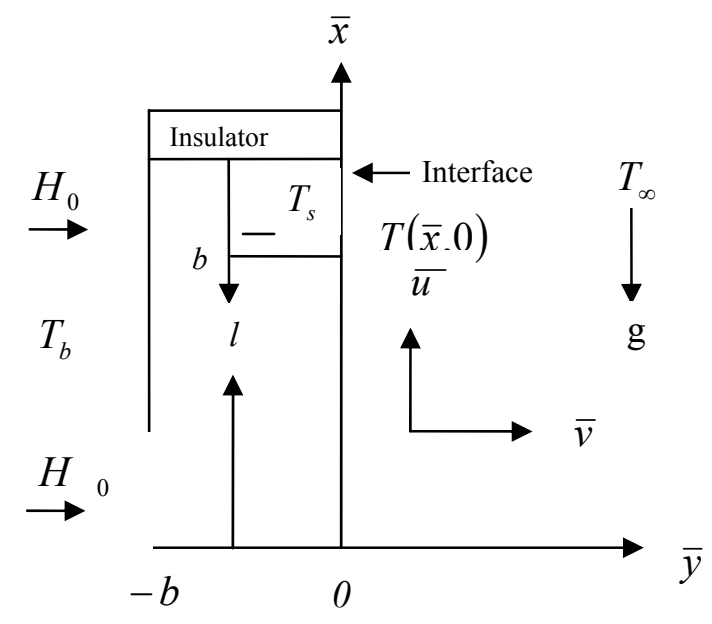

Fig. 1: Physical model and coordinate system

The governing equations of such flow under the Bousinesq approximation can be expressed within the boundary layer as $[8,13]$ :

$$
\begin{aligned}
& \frac{\partial \bar{u}}{\partial \bar{x}}+\frac{\partial \bar{v}}{\partial \bar{y}}=0 \\
& \bar{u} \frac{\partial \bar{u}}{\partial \bar{x}}+\bar{v} \frac{\partial \bar{u}}{\partial \bar{y}}=v \frac{\partial^{2} \bar{u}}{\partial \bar{y}}+g \beta\left(T_{f}-T_{\infty}\right)-\frac{\sigma H^{2}{ }_{0} \bar{u}}{\rho} \\
& \bar{u} \frac{\partial T_{f}}{\partial \bar{x}}+\bar{v} \frac{\partial T_{f}}{\partial \bar{y}}=\frac{k_{f}}{\rho C_{p}} \frac{\partial^{2} T_{f}}{\partial \bar{y}^{2}}-4 \Gamma\left(T_{f}-T_{b}\right)+\frac{v}{c_{p}}\left(\frac{\partial \bar{u}}{\partial \bar{y}}\right)^{2} \\
& +\frac{Q_{0}}{\rho C_{p}}\left(T_{f}-T_{\infty}\right)
\end{aligned}
$$

Where $\Gamma=\int_{0}^{\infty} K_{\lambda w}\left(\frac{\partial e_{b \lambda}}{\partial T_{f}}\right) d \lambda, \quad K_{\lambda w}=K_{\lambda}\left(T_{w}\right)$ is the mean absorption coefficient $e_{b \lambda}$ is Plank's function and $T_{f}$ is the temperature of the fluid at the boundary layer. Where kinematic viscosity $v$, Thermal expression coefficient $\beta$, Electrical conductivity $\sigma, C_{p}$ is the specific heat due to constant pressure. The boundary conditions are:

$$
\left.\begin{array}{l}
\bar{u}=\bar{v}=0, \quad T_{f}=T(\bar{x}, 0), \\
\frac{\partial T_{f}}{\partial \bar{y}}=\frac{k_{s}}{b k_{f}}\left(T_{f}-T_{b}\right) a t \bar{y}=0, \bar{x}>0 \\
\bar{u} \rightarrow 0, T_{f} \rightarrow T_{\infty} \text { at } \bar{y} \rightarrow \infty, \bar{x}>0
\end{array}\right\}
$$

We observe that the equations (1) to (3) together with the boundary conditions (4) are nonlinear partial differential equations. Equations (1) to (3) may now be nondimensionalized by using the following dimensionless dependent and independent variables:

$$
\left.\begin{array}{rl}
x & =\frac{\bar{x}}{l}, y=\frac{\bar{y}}{l}, u=\frac{\bar{u} l}{v} G r^{-1 / 2}, \\
v & =\frac{\bar{v} l}{v} G r^{-1 / 4}, \theta=\frac{T-T_{\infty}}{T_{b}-T_{\infty}} \\
G r & =\frac{g \beta l^{3}\left(T_{b}-T_{\infty}\right)}{v^{2}}, \\
M & =\frac{\sigma H_{0} l^{2}}{\mu} G r^{-1 / 2}, \\
R & =\frac{4 \Gamma l^{2}}{v} G r^{-1 / 2}
\end{array}\right\}
$$

Where $v=(\mu / \rho)$ is the kinematic viscosity, $G r$ is the Grashof number and $\theta$ is the nondimensional temperature. Using equation (5) in equations (1) to (3), we can obtain the governing equation in a dimensionless form as:

$$
\begin{aligned}
& \frac{\partial u}{\partial x}+\frac{\partial v}{\partial y}=0 \\
& u \frac{\partial u}{\partial x}+v \frac{\partial u}{\partial y}+M u=\frac{\partial^{2} u}{\partial y^{2}}+\theta \\
& u \frac{\partial \theta}{\partial x}+v \frac{\partial \theta}{\partial y}=\frac{1}{\operatorname{Pr}} \frac{\partial^{2} \theta}{\partial y^{2}}-R(\theta-1) \\
& +N\left(\frac{\partial u}{\partial y}\right)^{2}+Q \theta
\end{aligned}
$$

The corresponding boundary conditions are:

$$
\begin{aligned}
& u=v=0, \theta-1=p \frac{\partial \theta}{\partial y} \quad \text { at } \quad y=0, x>0 \\
& u \rightarrow 0, \theta \rightarrow 0 \quad \text { at } y \rightarrow \infty, x>0
\end{aligned}
$$

Here $M=\left(\sigma H_{0}{ }^{2} l^{2} / \mu\right) G r^{-1 / 2}$ is the magnetic parameter, $R=\left(4 \Gamma l^{2} / v\right) G r^{-1 / 2}$ is the radiation parameter, $\operatorname{Pr}=\left(\mu C_{p} / k_{f}\right)$ is the Prandtl number $N=\left(v^{2} G r / C_{p} l^{2}\left(T_{b}-T_{\infty}\right)\right)$ is the viscous dissipation parameter and $Q=\left(Q_{0} l^{2} / \mu C_{p}\right) G r^{-1 / 2}$ is the heat 
generation parameter and $p=\left(k_{f} / k_{s}\right)(b / l) G r^{1 / 4}$ is a conjugate conduction parameter. The value of the conjugate conduction parameter $p$ depends on $(b / l),\left(k_{f} / k_{s}\right)$ and $G r$ but each of which depends on the types of considered fluid and the solid. Therefore in different cases $p$ is different but not always a small number. In the present analysis we have taken $p=1$. The steam function and similarity variable and the dimensionless temperature are considered in the following form to solve the equations (7) and (8) and for the boundary conditions described in (9)

$$
\begin{aligned}
& \psi=x^{4 / 5}(1+x)^{-1 / 20} f(x, \eta), \\
& \eta=y x^{-1 / 5}(1+x)^{-1 / 20}, \\
& \theta=x^{1 / 5}(1+x)^{-1 / 5} h(x, \eta)
\end{aligned}
$$

Where $\psi$ is the dimensionless stream function which is related to the velocity components such as $u=\partial \psi / \partial y$ and $v=-\partial \psi / \partial x, \eta$ is the similarity variable and $h(x, \eta)$ is a dimensionless temperature. By substituting (10) in equations (7) and (8) and the boundary condition then we obtain the transformed equations:

$$
\begin{aligned}
& f^{\prime \prime}+\frac{16+15 x}{2 Q(1+x)} f^{\prime \prime}-\frac{6+5 x}{10(1+x)} f^{\prime 2} \\
& -M x^{2 / 5}(1+x)^{1 / 10} f^{\prime}+h=x\left(f^{\prime} \frac{\partial f^{\prime}}{\partial x}-f^{\prime \prime} \frac{\partial f}{\partial x}\right. \\
& \frac{1}{\operatorname{Pr}} h^{\prime \prime}+\frac{16+15 x}{20(1+x)} f h^{\prime}-\frac{1}{5(1+x)} f^{\prime} h-R x^{2 / 5} \\
& (1+x)^{1 / 10} h+R x^{1 / 5}(1+x)^{3 / 10}+N x f^{\prime \prime 2} \\
& +Q x^{2 / 5}(1+x)^{1 / 10} h=x\left(f^{\prime} \frac{\partial h}{\partial x}-h^{\prime} \frac{\partial f}{\partial x}\right)
\end{aligned}
$$

The boundary condition (9) become

$$
\left.\begin{array}{l}
f(x, 0)=f^{\prime}(x, 0)=0, h^{\prime}(x, 0)=-(1+x)^{1 / 4} \\
+x^{1 / 5}(1+x)^{1 / 20} h(x, 0) \text { aty }=0 \\
f^{\prime}(x, \infty) \rightarrow 0, h(x, \infty) \rightarrow \text { aty } \rightarrow \infty
\end{array}\right\}
$$

The set of equation (11) and (12) together with the boundary condition (13) are solved numerically by applying Implicit finite difference method with Keller-Box [14] Scheme. Detailed numerical method is given in the book "Physical and computational aspects of convective heat transfer" written by Cebeci and Bradshaw [15]. In practical point of view, it is important to calculate the values of the skin friction co-efficient. This can be written in the dimensionless form as

$$
C_{f}=\left(G r^{-3 / 4} l^{2} / \mu v\right) \tau_{w}
$$

Where $\tau_{w}=\mu\left(\frac{\partial \bar{u}}{\partial \bar{y}}\right)_{\bar{y}=0}$ is the shearing stress.

Thus the local skin friction co-efficient is

$$
C_{f x}=x^{2 / 5}(1+x)^{-3 / 20} f^{\prime \prime}(x, 0)
$$

and the numerical value of the surface temperature distribution are obtained from the relation

$$
\theta(x, 0)=x^{1 / 5}(1+x)^{-1 / 5} h(x, 0)
$$

We have discussed the velocity profiles and temperature distributions for various values of Prandtl number, magnetic parameter, radiation parameter heat generation parameter and viscous dissipation for the present investigation.

\section{Results and Discussion}

The numerical results for the velocity and the temperature are illustrated in Figs. 2-6 and the skin friction and surface temperature are depicted in Figs. 7-11. The values of prandtl number are taken to be $0.733,0.900,1.000$ and 1.446 which correspond to air, ammonia, steam and water respectively. The detailed numerical solutions have been obtained for a wide range of values of the parameters as $M=0.10-1.00$, $R=0.001-0.05, Q=0.01-0.10$ and $N=0.01-1.00$.

The numerical values of velocity and temperature against $\eta$ are obtained from the solution of the equations (11) and (12) with the boundary condition (13) for different values of magnetic parameter $M$ when $P r=0.733$ $R=0.001 Q=0.01$ and $N=0.01$ and are illustrated in Fig. 2(a) and Fig. 2(b), respectively. The magnetic field acting along the horizontal direction and exert a pull on flowing fluid particle as well as create retard forces which 
oppose the motion of the fluid. As a result, the velocity of the fluid decreases with the increasing value of magnetic parameter is shown in figure 2(a).

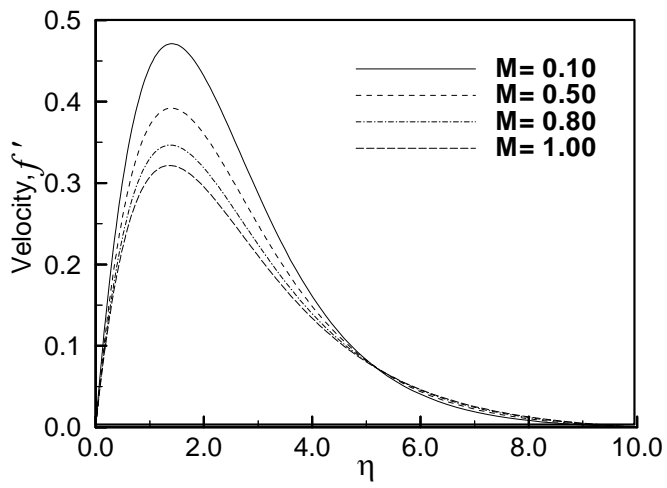

(a)

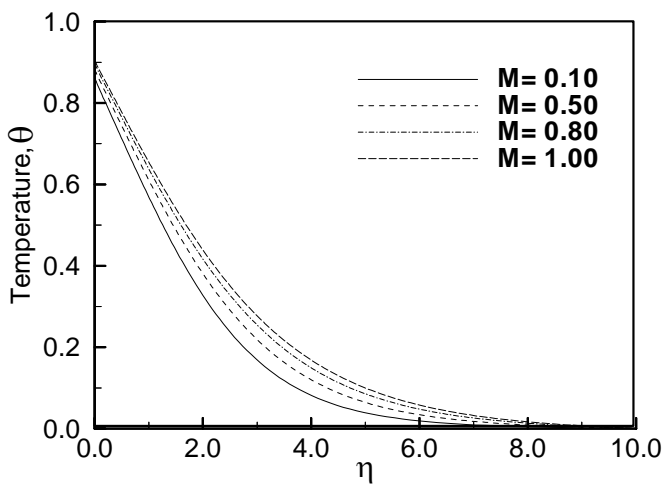

(b)

Fig. 2: (a) Variation of velocity and (b) Variation of temperature against $\eta$ for varying of $M$ with $P r$ $=0.733, R=0.001, Q=0.01$ and $N=0.01$

For each value of $M$, the velocity is zero at the boundary wall and then increase to maximum value as $\eta$ increases and finally approach to zero. Moreover, we have seen that the velocity profiles meet together after certain value of $\eta$ and cross the side. This is because, the gradient of decreasing of velocity increases with the increasing of magnetic parameter. In Fig. 2 (b), it can be seen that the temperature increases within the boundary layer for the increasing values of magnetic parameter $M$ due to interaction of applied magnetic field and fluid particles. Moreover, the temperature decreases monotonically with increasing of $\eta$ for a particular value of $M$. The maximum values of the temperature are $0.8613,0.8819$,
0.8955 and 0.9038 for $M=0.10,0.50,0.80$ and 1.00 , respectively. Each of which occurs at the surface of the plate. Here the magnetic field works to retard the fluid motion as well as increase the temperature and the thickness of the boundary layer.

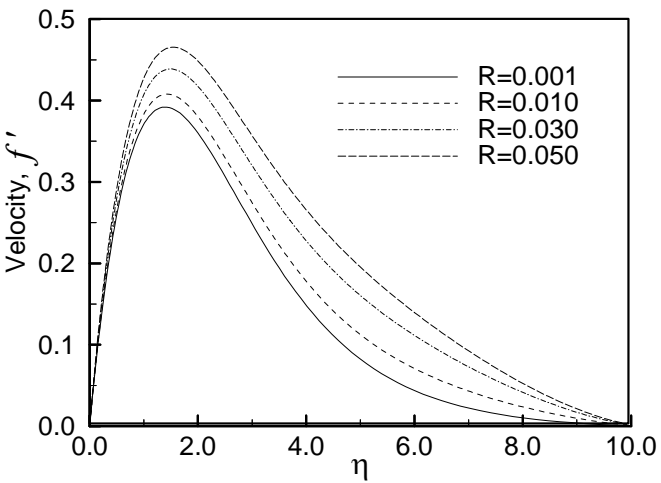

(a)

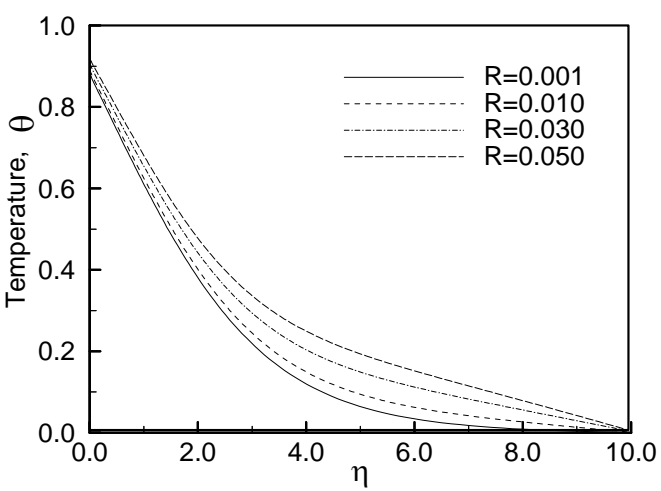

(b)

Fig. 3: (a) Variation of velocity and (b) variation of temperature against $\eta$ for varying of $R$ with $P r=0.733, M=0.50, Q=0.01$ and $N=0.01$.

The variation of the velocity and the temperature distributions with $\eta$, respectively for some selected values of the radiation parameter $R$ together with a certain value of Prandtl number $P r$, magnetic parameter $M$, heat generation parameter $Q$ and viscous dissipation parameter $N$ presented in Fig. 3 (a) and Fig. 3 (b), respectively. Fluid absorbed heat while radiation imitates from the heated plate, as a result the motion and the temperature of the fluid increases with in the flow region. That's why the velocity and the temperature increase with the increasing of $\mathrm{R}$. It can be noted that, near the surface of the flat plate, velocity increases by a long way and becomes maximum. It then decreases along 
horizontal direction. The maximum values of the velocity are $0.3920,0.4079,0.4387$ and 0.4655 for $R=0.001,0.010,0.030$ and 0.050 at $\eta=1.3827,1.4279,1.4741$ and 1.5214. From Fig. 3 (a) and also numerical values we seen that the velocity profiles shift upward and the position of the peak velocity moves toward the boundary layer for the increasing $R$. As the temperature of the fluid increases with the increasing of radiation as shown in Fig. 3(b), accordingly the thickness of the boundary layer increases observed in Fig. 3(b).

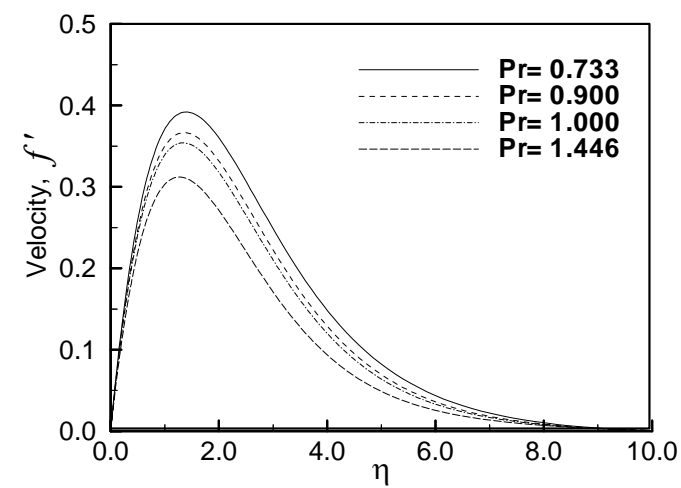

(a)

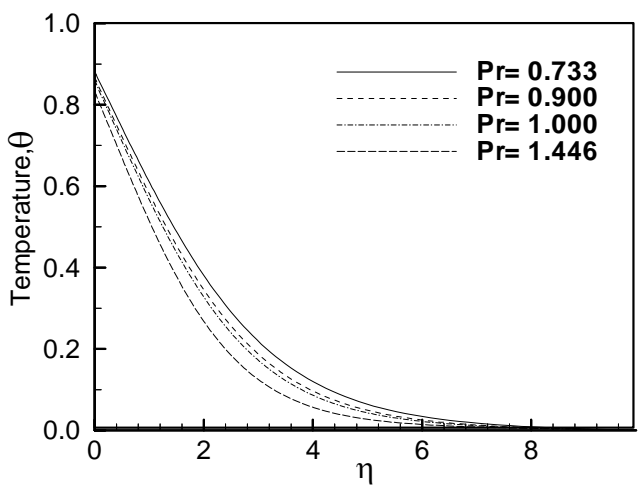

(b)

Fig. 4: (a) Variation of velocity and (b) variation of temperature against $\eta$ for varying of $\operatorname{Pr}$ with $M=0.50, R=0.001, Q=0.01$ and $N=0.01$.

Numerical values of the velocity and temperature with respect to $\eta$ are depicted graphically in Fig.4(a) and Fig. 4 (b), respectively for different values of Prandtl number $\operatorname{Pr}$ with magnetic parameter $M=0.50$, radiation parameter $R=0.001$, heat generation parameter $Q=0.01$ and viscous dissipation $N=0.01$. The increasing value of $\operatorname{Pr}$ increase viscosity of the fluid. Viscosity increase means that the density of the fluid increase which results fluid does not move freely. It can be seen that the velocity decreases gradually and the peak velocity moves towards the interface for the increasing $P r$. Moreover, the velocity is zero at the wall and increases to the peak as $\eta$ increases and finally approaches to zero. These are expected behavior because it supports the no-slip condition at the wall and the fluid motion outside the boundary layer. Fig. 4 (b) shows that the temperature distribution over the whole the boundary layer decreases due to the increase of Pr. It agree the physical fact that the temperature at the solid fluid interface is reduced because, temperature at the plate considered constant and thermal boundary layer thickness as well as velocity decrease with the increasing of $\mathrm{Pr}$.

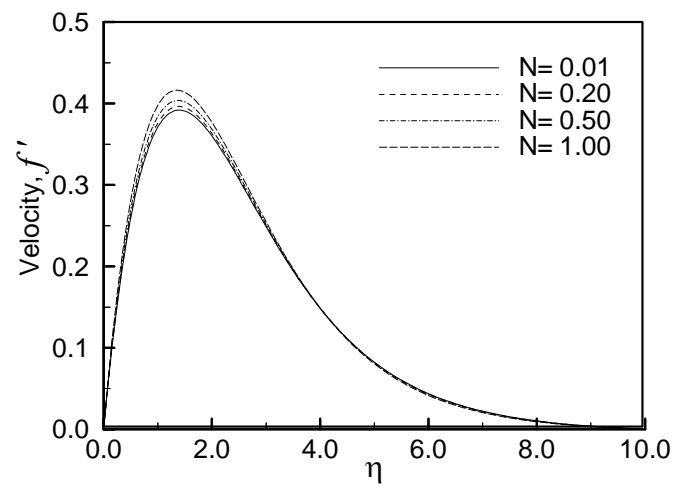

(a)

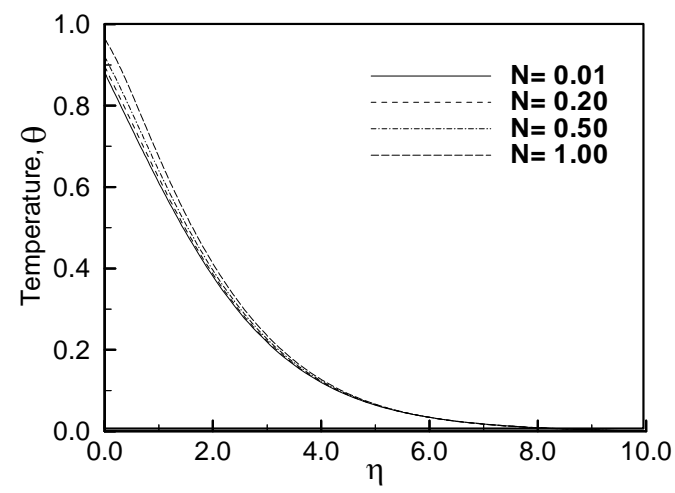

(b)

Fig.5: (a) Variation of velocity and (b) variation of temperature against $\eta$ for varying of $N$ with $\operatorname{Pr}=0.733, M=0.50, R=0.001$ and $Q=0.01$.

Fig. 5 (a) and Fig. 5(b), respectively illustrates the effects of $N$ on velocity and temperature while $P r=0.733, M=0.50, R=0.001$ and $Q=0.01$. From Fig. 5(a) conclude that the velocity profiles increases slightly due to the 
increase of viscous dissipation parameter $N$ along the $\eta$ direction and also we have seen that near the surface of the plate the velocity is increases to maximum with increase of viscous dissipation parameter $N$ then after the peak position start to decrease and finally approaches to zero. The maximum values of the velocities are $0.3920,0.3964,0.4037$ and 0.4163 which occurs for $N=0.01,0.20,0.50$ and 1.00 respectively. On the other hand from Fig. 5(b), we have seen that the same result is holds for temperature distributions within the boundary layer due to increasing of viscous dissipation parameter $N$.

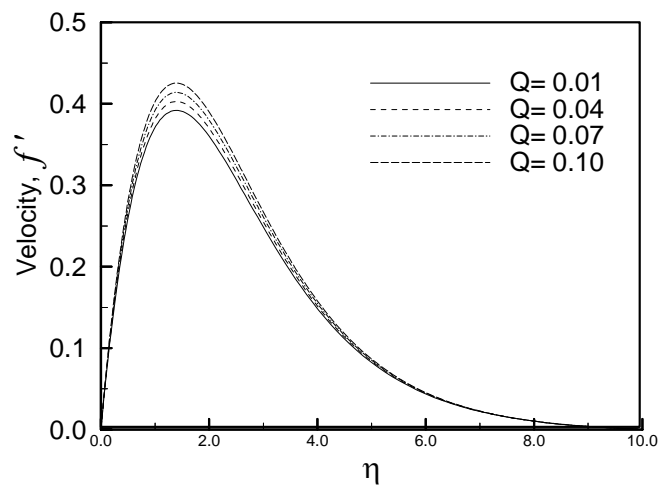

(a)

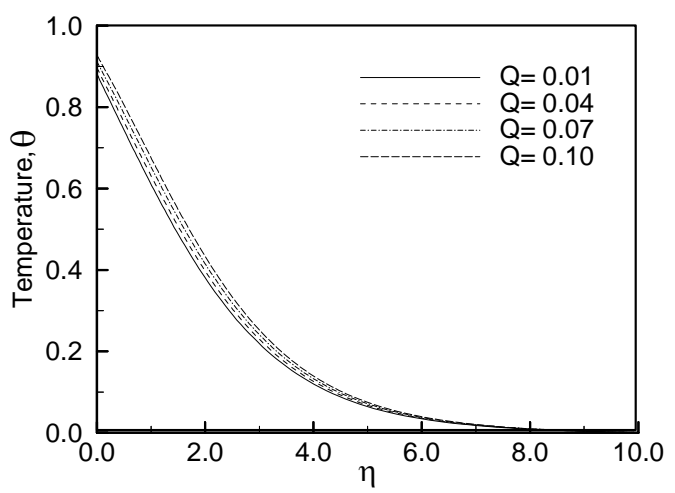

(b)

Fig.6: (a) Variation of velocity and (b) variation of temperature against $\eta$ for varying of $Q$ with $\operatorname{Pr}=0.733, M=0.50, R=0.001$ and $N=0.01$.

The maximum value of temperature are 0.8819 , $0.8961,0.9199$ and 0.9639 for viscous dissipation parameter $N=0.01,0.20,0.50$ and 1.00 , respectively. Each of which occurs at the surface of the plate. This is because; the increased value of dissipation parameter $N$ produces heat and also dissipates viscosity of fluid.

The effects of heat generation on velocity and temperature with respect to $\eta$ are shown in Fig.6 (a) and 6(b), respectively. It is observed from figures, the increased value of heat generation parameter $Q$ leads to increase the velocity and temperature profiles within the boundary. Moreover, the velocity profile shifts upward for the increasing $Q$ and temperature profile also moves up with a rise in heat generation parameter $Q$ while the values of controlling parameter are kept fixed.

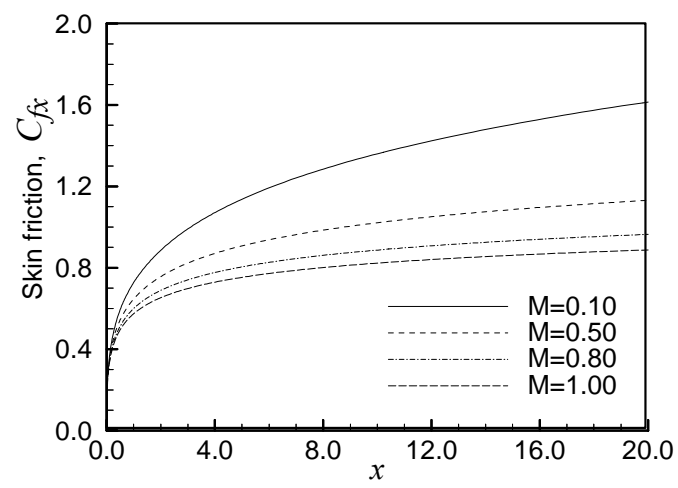

(a)

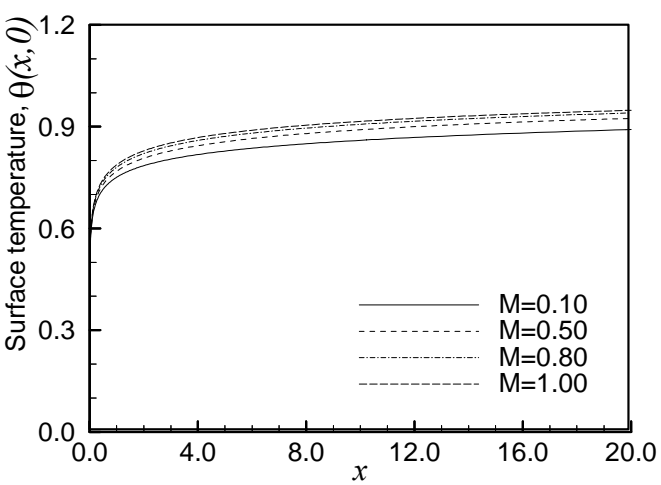

(b)

Fig.7: (a) variation of skin friction and (b) variation of surface temperature against $x$ for varying of $P r$ with $M=0.50, R=0.001, Q=0.01$ and $N=0.01$.

Fig. 7(a) and Fig. 7(b), reveals that the skin friction coefficient and the surface temperature for some selected values of $M$ with $P r=0.733$, $R=0.001, Q=0.01$ and $N=0.01$. The increased value of $M$ leads to a decrease of the skin friction coefficient and increase surface temperature on the plate. This is because, due to the interaction between applied magnetic 
field and fluid field. The magnetic field retards the fluid motion and reduces the skin friction coefficient and produce temperature at the solid fluid interface.

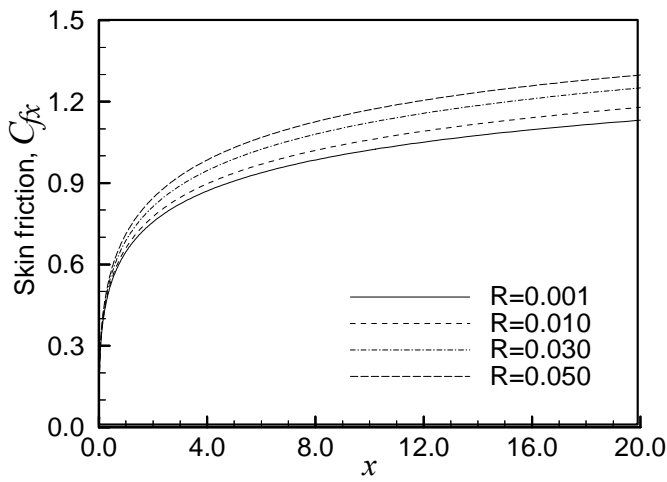

(a)

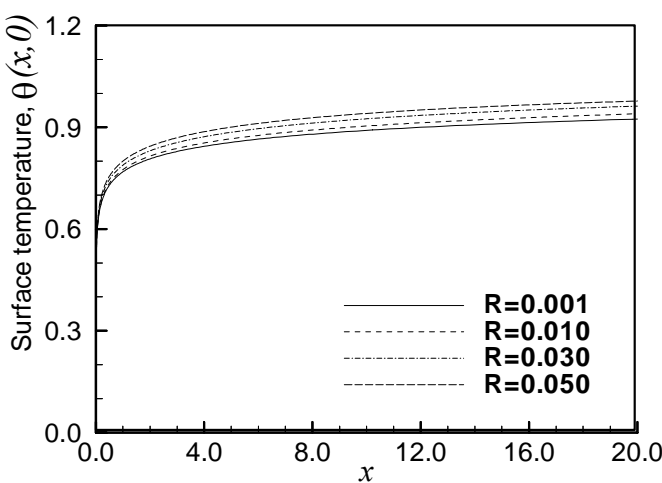

(b)

Fig.8: (a) variation of skin friction and (b) variation of surface temperature against $\mathrm{x}$ for varying of $R$ with $\operatorname{Pr}=0.733, M=0.50, Q=0.01$ and $N=0.01$.

The variation of the local skin friction coefficient $C_{f x}$ and local surface temperature $\theta(x, 0)$ for different values of $R$ associated with $P r=0.733, M=0.50, Q=0.01$ and $N$ $=0.01$ are illustrated in Fig. 8(a) and Fig. 8(b), respectively. Radiation increases the fluid motion as mentioned in Fig. 3(a) and increases the shear stress at the wall, for which local skin friction increase with the increasing of $\mathrm{R}$. This phenomenon demonstrated in Fig. 8(a). The increased value of the radiation parameter increases the temperature (Fig. 3(b)) which after increase the surface temperature along the $\mathrm{x}$-direction. Moreover, surface temperatures depend on temperature variation. As the temperature of fluid increase with in the boundary layer, the surface temperature also increases.

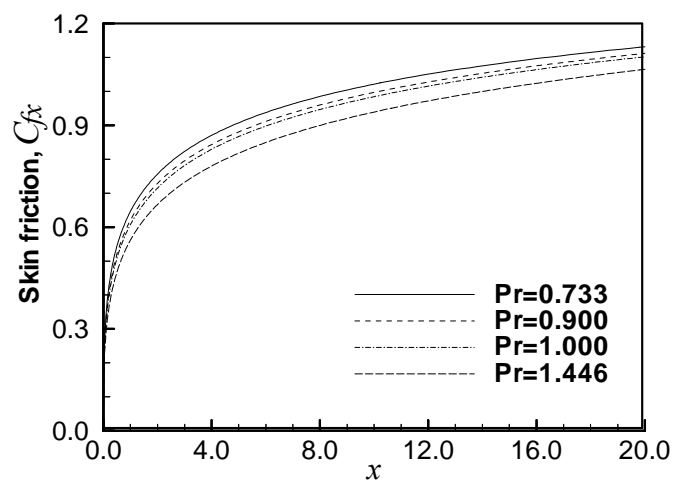

(a)

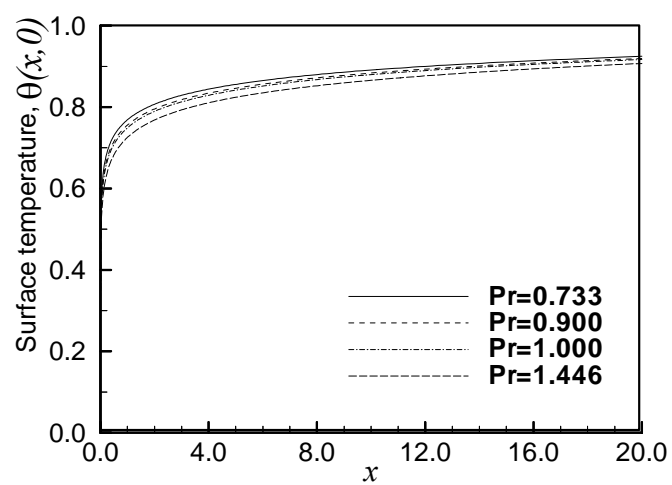

(b)

Fig.9: (a) variation of skin friction and (b) variation of surface temperature against $\mathrm{x}$ for varying of $\mathrm{Pr}$ with $M=0.50, R=0.001, Q=0.01$ and $N=0.01$.

The effects of Prandtl number on the skin friction $C_{f x}$ and surface temperature $\theta(x, 0)$ with the increasing of axial distance $x$ for the selected values of magnetic parameter, radiation parameter, heat generation parameter and viscous dissipation parameter is shown in Fig. 9(a) and Fig. 9(b), respectively. The values of $\mathrm{Pr}$ are proportional to the viscosity of the fluid. So the increased values of $P r$ decrease the velocity and temperature of the fluid within the boundary layer, as a result corresponding skin friction coefficient and surface temperature also decreases as shown in Fig.9(a) and Fig. 9(b). Moreover, for a particular value of $\mathrm{Pr}$ the local skin friction coefficient and surface temperature increases monotonically due to the increasing of $x$. 
Fig. 10(a) and Fig. 10(b) illustrates the local skin friction coefficients and surface temperature for different values of $N$ with increasing of $x$ and controlling parameters $\mathrm{Pr}=$ $0.733, R=0.001, M=0.50$ and $\mathrm{Q}=0.01$.

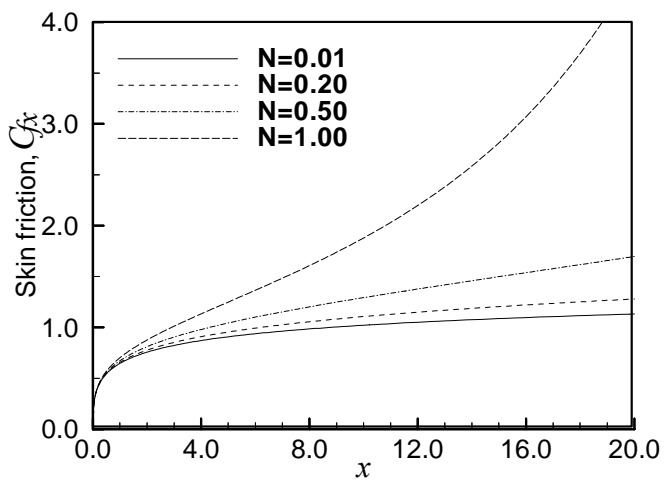

(a)

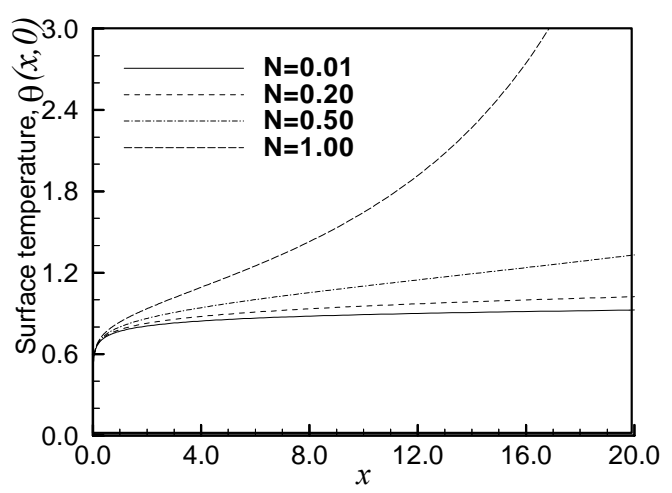

(b)

Fig.10: (a) variation of skin friction and (b)variation of surface temperature against x for varying of $N$ with $P r=0.733, M=0.50, R=0.001$ and $Q=0.01$.

The velocity and the temperature of the fluid increase with the increasing of $N$ that has been shows in Fig.5 (a) and 5(b), respectively accordingly corresponding skin friction and surface temperature increase associated with the increasing of $N$. It is also observed that the skin friction and surface temperature change rapidly with small change of viscous dissipation parameter.

Fig.11 (a) presents the variation of skin friction coefficient for varying of heat generation parameter. The skin friction coefficient increases associated with the increasing of $Q$ due to the heat generation effects, which accelerates the fluid flow. Similar situation is observed in Fig.11 (b) for the case of surface temperature distribution within the boundary layer for increasing heat generation parameter.

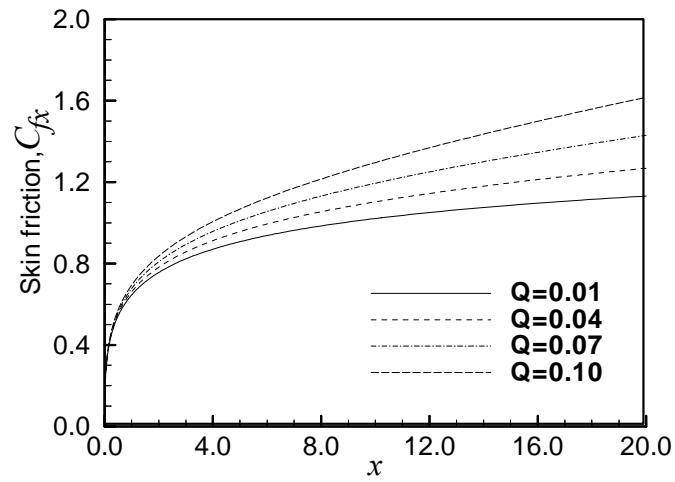

(a)

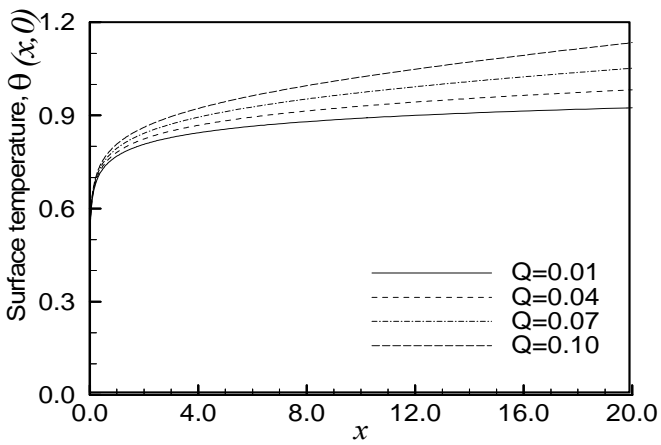

(b)

Fig.11: (a) variation of skin friction and (b)variation of surface temperature against x for varying of $Q$ with $P r=0.733, M=0.50, R=0.001$ and $N=0.01$.

\section{Conclusion}

In this analysis the effect of radiation and viscous dissipation on magnetohydrodynamic (MHD) natural convection flow along a vertical flat plate in presence of heat generation has been investigated for some selected values of pertinent parameters including magnetic parameter, radiation parameter, Prandtl number, heat generation parameter and viscous dissipation parameter. From the present investigation, it may be concluded that the velocity of the fluid and the skin friction at the interface decrease with the increasing magnetic field and Prandtl number while they increase with the increasing of radiation parameter, heat generation parameter and viscous dissipation parameter. The temperature of the fluid and also the surface temperature increases with the increasing magnetic field, radiation parameter, 
heat generation parameter and viscous dissipation but decrease for Prandtl number.

\section{References}

[1] M. E. Sparrow, R. D. Cess, "Effect of magnetic field on free convection heat transfer", Int. Journal Heat and Mass Transfer ,Vol.3, No.4, 1961, pp.267-274.

[2] K. R. Sing, T. G. Cowling, "Thermal conduction in magnetohydrodynamics", Journal of Mech. Appl. Math., Vol.16, 1963, pp.1-5.

[3] N. Riley, "Magnetohydrodynamic free convection", Journal of Fluid Mech., Vol.18, 1964, pp-577-586.

[4] H. S. Takhar, V. M. Soundalgekar, "Radiation effects on MHD free convection flow of a gas past a simi- infinite vertical plate", Applied Scientific Research, Vol.36, 1980, pp. 163-171.

[5] M. A. Hossain, M. A. Alim, "Natural convectionradiation interaction on boundary layer flow along a thin vertical cylinder", Heat and Mass Transfer, Vol. 32, No.6, 1997, pp.515-520.

[6] M. A. Hossain, M. A. Alim, D.A.S Ress, "The effect of radiation on free convection from a porous vertical plate", Int Journal Heat and Mass Transfer, Vol.42, No.1, 1999, pp.181-191.

[7] M. A. Seddeek, "Thermal radiation buoyancy effects on MHD free convective heat generating flow over an accelerating permeable surface with temperature dependent viscosity", Canadian Journal of Phys., Vol.79, No.4, 2001, pp. 725-732.

[8] Abd El-Naby, Elsayed M. E.Elbarbaryand Nader Y. Abdelazem, "Finite difference solution of radiation effect on MHD unsteady free convection flow over a vertical plate variable surface temperature", Journal of Applied Mathematics, Vol.2003, No.2, 2003, pp.65-86.

[9] M. M. Molla, M. A. Taher, M. M. K. Chowdhury, M. A. Hossain, "Magnetohydrodynamic natural convection flow on a sphere in presence of heat generation effect", Nonlinear analysis modeling and control, Vol.10, No.4, 2005, pp.349-363.

[10] M. M. Molla, M. A. Taher, M. A. Hossain, "Magnetohydrodynamic natural convection flow on a sphere with uniform heat flux in presence of heat generation", Acta Mech. Vol.186, No.1-4, 2006, pp.75-86.

[11] M. M. Alam, M. A. Alim, M. M. K. Chowdhury, "Viscous dissipation on MHD natural convection flow over a sphere in presence of heat generation", Nonlinear Analysis, Modelling and control, Vol.12, No.4, 2007, pp. 447-459.

[12] A. A. Mamun, Z. R. Chowdhury, M. A. Azim, M. A. Maleque, "Conjugate heat transfer for a vertical flat plate with heat generation effect", Nonlinear analysis modeling and control, Vol.13, No.2, 2008, pp.1-11.

[13] A. A. Mamun, Z. R. Chowdhury, M. A. Azim, M. M. Molla, "MHD-conjugate heat transfer analysis for a vertical flat plate in presence of viscous dissipation and heat generation", Int. communications in heat and mass transfer, Vol.35, No.10, 2008, pp-12751280.

[14] H. B. Keller, "Numerical methods in the boundary layer theory", Annu. Rev. Fluid Mech., Vol.10, No.1, 1978, pp. 417-433.

[15]T. Cebeci, P. Bradshow, Physical computational aspects of convective heat transfer, Springer, New York, 1984. 